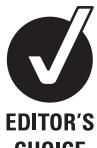

CHOICE

${ }^{1}$ University of Missouri Center for Health Care Quality, Department of Health

Management and Informatics, Columbia, Missouri, USA; ${ }^{2}$ Harry

S Truman Memorial Veterans Hospital, University of Missouri Sinclair School of Nursing, Columbia, Missouri, USA

Correspondence to: Dr D S Wakefield, Center for Health Care Quality (CHCO), MA120 Medical Sciences Bld. DC375.00, One Hospital Drive, Columbia, M0 65212, USA; wakefielddo@health.missouri. edu

Accepted 27 April 2009

\title{
Are verbal orders a threat to patient safety?
}

\author{
D S Wakefield, ${ }^{1}$ B J Wakefield ${ }^{2}$
}

\begin{abstract}
Background: The use of verbal orders has been identified as a potential contributor to poor quality and less safe care. As a result, many organisations have encouraged changing the verbal orders process and/or reducing/eliminating verbal orders altogether (Joint Commission (2005), Institute of Medicine (2001), Leapfrog organisation, Institute of Safe Medication Practices). Ironically there is a paucity of research evidence to support the widespread concern over verbal order.
\end{abstract}

Aims: This paper describes the very limited existing research on verbal orders, presents a model of verbal order use identifying potential error trigger points and suggests a verbal order research agenda in order to better understand the nature and extent of the potential patient care safety threat posed by verbal orders.

Verbal orders (VO) represent a commonly used and important communication mechanism in the patient care environment. Whether given face to face, by telephone or by other voice transmission devices, VO can facilitate up-to-the minute communication of a patient's clinical status, laboratory and other information, and result in more timely clinical decision-making. While not well studied, VO may represent $20 \%$ or more of all inpatient orders $^{1-3}$ and are commonly used when prescribers (ie, physician, nurse practitioner (NP), physician's assistant (PA)) are unable or unwilling to write in the medical record or electronically enter orders using a computerised provider order entry (CPOE) system. Thus, there are both appropriate and inappropriate uses of VO. For example, the use of face-to-face $\mathrm{VO}$ is clearly necessary when the prescriber is in the middle of a procedure or medical emergency, and it is impractical to stop patient care to write a patient care order. Likewise, telephone VO may be necessary if the prescriber is not physically present when a new patient care order is needed (eg, at night). There are also instances when VO should not be used, that is, complex chemotherapy or do-not-resuscitate orders. In between these indicators of appropriate and inappropriate $\mathrm{VO}$ use is a great variation in their appropriate use during other types of patient care activities (eg, patient rounds, interdisciplinary team meetings, other non-emergent patient care situations or teaching healthcare learners). Of particular concern is the extent to which face-toface VO may become routine and used as a convenience rather than a necessity, thus replacing prescribers' writing or electronically entering patient care orders.

This paper briefly describes the existing research on $\mathrm{VO}$, presents a process model of $\mathrm{VO}$ use that identifies potential error trigger points and suggests a $\mathrm{VO}$ research agenda. For ease of presentation, we will specifically use the terms "face-to-face verbal order" and "telephone order" to distinguish when we are discussing specific types of verbally communicated patient care orders. Otherwise the acronym VO will include all telephone or face-toface patient care orders that: (a) the ordering prescriber (eg, physicians, NPs, PAs) communicates verbally (eg, by telephone or face-to-face); (b) require subsequent transcription to the patient's medical record (either paper-based or electronically) by those individuals approved to receive $\mathrm{VO}$; and (c) require the prescriber to subsequently review and sign the transcribed order.

\section{VO LITERATURE}

\section{Research literature}

Despite the perception of their potential for patient harm, the indications for using $\mathrm{VO}, \mathrm{VO}$ policies and procedures, and the extent of $\mathrm{VO}$ use have not been studied in depth. ${ }^{2-4}$ The VO literature consists primarily of non-systematic and anecdotal evidence of the relationship between VO utilisation and actual or potential patient harm. ${ }^{56}$ To our knowledge, the only large-scale study of hospital VO polices is a 1990 report of a survey of nursing and pharmacy leaders' self-report of selected features of their hospitals' VO policies. The only study specifically looking at errors associated with $\mathrm{VO}$ was conducted in an inpatient paediatric setting in the mid 1990s, and had the counterintuitive finding of a fourfold decreased risk of error associated with verbal as compared with handwritten orders. ${ }^{1}$ A more recent systematic review of the literature assessing evidence of the error risk associated with $\mathrm{VO}$ also found only the one aforementioned study and, based on the lack of research in this area, concluded that despite the "common-sense" of limiting VO, there is no empirical support for adopting changes in $\mathrm{VO}$ policies. ${ }^{4}$ Recent work by Kaplan et al ${ }^{2}$ provides the most detailed hospital-wide analysis of VO utilisation. In this study of $\mathrm{VO}$ occurrence following implementation of a CPOE system, the authors found a decrease in VO rates from $23 \%$ to $10 \%$, a marked reduction in unsigned VO (43\% to $9 \%$ ), and significant variation in $\mathrm{VO}$ rates and content among different clinical services. Finally, Wakefield et al use VO data from one hospital to describe factors adding to the $\mathrm{VO}$ complexity. ${ }^{3}$

\section{Practice literature}

Despite the paucity of research evidence supporting either the use or elimination of $\mathrm{VO}$, it is widely believed that $\mathrm{VO}$ represent a threat to patient safety. This is most clearly evidenced by specific recommendations from the Joint Commission, the National Quality Forum (NOF) and others 


\section{Box 1 Examples of verbal orders having patient care quality and safety implications}

\section{Case 1}

- "A verbal order for flutamide was misheard as thalidomide. Errors are more likely with these sound-alike products because both may be used to treat prostate cancer."17

\section{Case 2}

- "A physician who intended to prescribe $40 \mathrm{mEq}$ of potassium chloride to be given IV over an hour instead gave a verbal order for '40 of K.' The order was misunderstood and the patient received $40 \mathrm{mg}$ of vitamin $\mathrm{K}$ intravenously." 18

\section{Case 3}

- "An emergency room nurse thought the physician stated that a patient was to receive '1 and 1/2 teaspoons' of Zithromax, which was given. In checking the written order, the dose was noted for $1 / 2$ teaspoon."19

\section{Case 4}

- " ... during a code situation, a verbal order for amiodarone was called into the pharmacy-no dose was specified. The pharmacist mistakenly dispensed amrinone, subsequently realised the error, and was able to get to the emergency department before the drug was administered."20

\section{Case 5}

- "Due to fluid restrictions, a physician gave a verbal order for a double-strength solution of magnesium sulfate to be administered at $2 \mathrm{~g} /$ hour. The nurse forgot to transcribe the verbal order and did not re-label the single-strength bag to which she had added additional magnesium sulfate. The change-of-shift report was hurried due to an emergency Cesarean section. The oncoming nurse subsequently increased the rate of infusion because she was unaware the patient was receiving a double-strength solution. The patient developed signs of magnesium toxicity..." 21

\section{Case 6}

- "A patient admitted to the intensive care unit with septic shock requiring vasopressors appears to have suffered a myocardial infarction (MI) .... the cause of the MI was likely related to a log increase in the dose of vasopressin because of a prescribing error....The vasopressin order was incorrectly written by a resident physician after he received a verbal order from his supervising critical care fellow."22

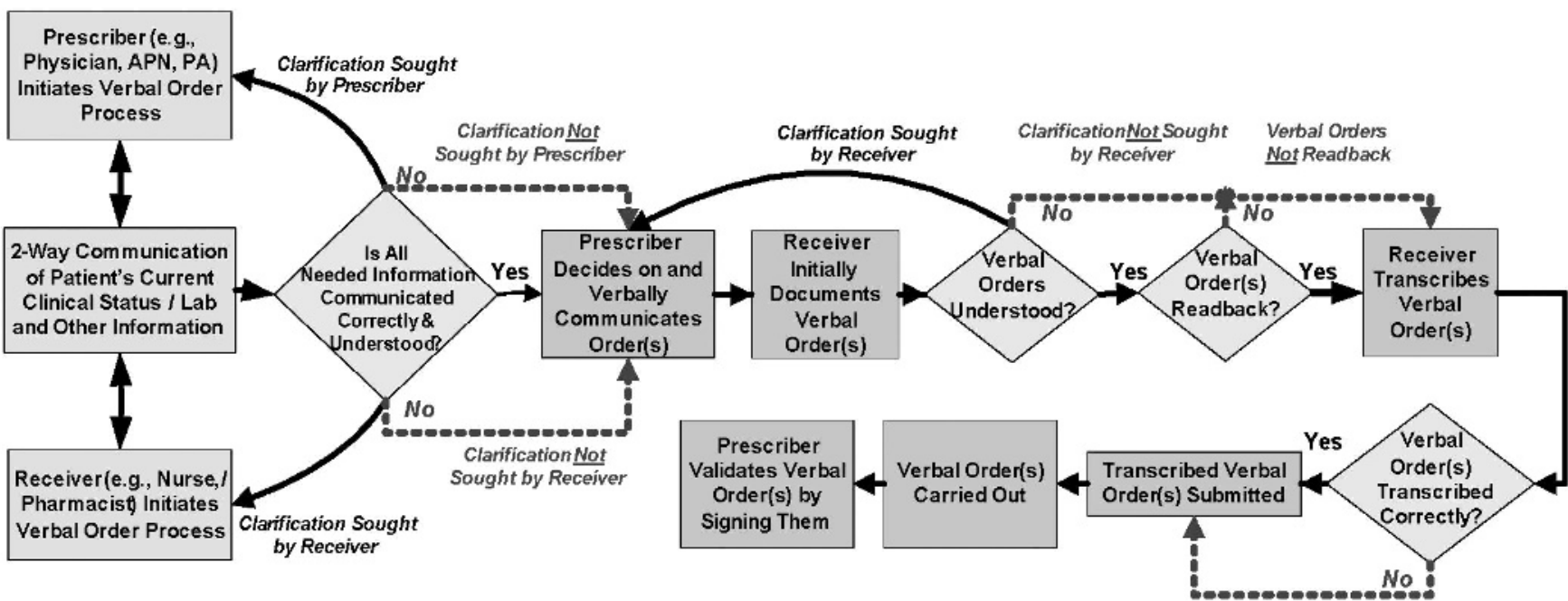

Why Verbal Communication Mav Be Incorrect or Misunderstood.

1) Misspeaking

2) Background noise, hearing difficulties

3) Muffled voices, accents, mispronunciations

4) Not familiar with patient's condition / status

5) Fails to get understanding of all other care

6) Confuses patients or patient data

7) Use of unapproved abbreviations or doses
Reasons Why Clarification / Readbacks Not Done:

1) Misunderstand sound -alike medications

2) Fear of appearing incompetent

3) Prescriber's response may be negative

4) Failure to recognise lack of understanding

5) Inadequate staffing for work load

6) Previous experience between prescriber and order receiver in working together
Causes of Verbal Order Errors

1) Incorrect communication of patient's status

2) Making the wrong decision

3) Failure to seeking clarifications

4) Failure to understanding and $/$ or read back verbal order

5) Transcription error

Figure 1 Verbal order process model (solid arrows indicate intended flow. Broken arrows indicate unintended flow. 
requiring "read-backs" and other strategies aimed at reducing or standardising how VO are communicated..$^{8-16}$ The reason for this concern is that there are a variety of human and environmental factors ${ }^{12}{ }^{13}$ (eg, fatigue, workload, sound-alike medications, background noise, accents, dialects and different pronunciations), and a number of different individuals and steps involved in the VO process that may contribute to miscommunication, misunderstanding and transcribing errors. Box 1 provides examples of adverse events involving VO drawn from anecdotal reports in the literature that highlight several different types of problems associated with $\mathrm{VO}$.

Regardless of the reason $\mathrm{VO}$ are used, the potential for $\mathrm{VO}$ to be miscommunicated, misunderstood or incorrectly transcribed has received significant attention in practice. The vast majority of the available VO-related literature has focused on practical recommendations to limit $\mathrm{VO}$ use, and to standardise $\mathrm{VO}$ policies and practices to optimise $\mathrm{VO}$ communications, decision-making, understanding, transcription and execution. ${ }^{4-6}$ 8-16 Specific recommendations and suggestions can be categorised into seven general areas including specification of: (1) when VO should and should not be used; (2) who is allowed to give and receive the $\mathrm{VO}$; (3) what constitutes a $\mathrm{VO}$, and the related verbal content that must be documented; (4) limitations and prohibitions in the use of $\mathrm{VO}$; (5) authorisation and validation of the $\mathrm{VO}$; (6) strategies, techniques and specific actions to increase the clarity and effectiveness of the VO; and (7) requirements for periodic monitoring and evaluation of $\mathrm{VO}$ practices and compliance with organisational policies and procedures.

\section{Model of VO process}

Figure 1 depicts a VO model containing both intended process steps ("solid arrows"), and identifies points in the process where errors may occur with deviations from the intended process steps ("broken arrows"). Reasons why VO may be miscommunicated or misunderstood, and reasons why readbacks may not be performed are also listed. As shown in fig 1, the VO process may be initiated by either the prescriber or the receiver. An example of the latter would be a nurse detecting important changes in a patient's clinical condition and deciding to call the physician to share this information to determine whether any additional orders are needed. It should be noted that this initial communication between the prescriber (physician) and receiver (nurse) also has the potential to result in a miscommunication or misunderstanding of all of the verbally communicated patient clinical information necessary to make the correct decision about the specific order(s) that may be needed. That is, an incorrect $\mathrm{VO}$ may result from problems in the verbal (or written) communications preceding the VO. Examples of causes of VO-related errors are listed at the bottom of fig 1.

Verbally communicated orders may not be accurately heard or understood by the receiver (cases $1-3$, box 1 ). Ideally, and a potentially unique advantage of $\mathrm{VO}$, there would be the opportunity to seek immediate clarification and to "read back" the order when the VO is given. Case 4 in box 1 is an example of failure to clarify a VO. Readbacks allow for clarification about whether the receiver correctly heard and understood the order. Finally, there may be errors in either the initial order documentation (ie, writing down the order as it is given) and/

Box 2 Examples of potential verbal order (VO) research questions

1. Nature, extent and appropriateness of current $\mathrm{VO}$ use:

- What is the nature and variation in use of face-to-face or telephone verbal order in different types of care units (ie, critical care, medicine, surgery, paediatrics, psychiatry) and care settings (ie, acute care hospitals, ambulatory surgical centers, nursing homes)?

- What is the variation in the types of different face-to-face or telephone VO (ie, medication, laboratory, consultation requests...) made in different types of care units or care settings?

- Are there, and what are the, criteria used to define or determine when a face-to-face or telephone verbal order is deemed as being appropriate in different types of care settings?

- What are the trade-offs between the potential of VO to cause harm versus their potential to facilitate patient care processes?

2. Nature, extent and causal role of VO in medical error:

- What proportion of all medication-related errors (ie, prescribing, dispensing and administration) involve face-to-face and/or telephone V0?

- What proportion of all face-to-face and/or telephone VO result in a medication-related prescribing, dispensing or administration error, and/ or patient harm?

- For all face-to-face and/or telephone V0 associated with an error, to what extent was the cause of the error related to non-V0 communication errors, misunderstanding of what was being communicated or problems in transcribing the order prior to the execution of the VO?

- How do the risk factors for communication errors (ie, language skills, background noise levels, knowledge of the patient) vary between face-to-face and telephone VO?

- What steps in the VO process (ie, communication of patient status, understanding, reading back, transcribing, etc) are more prone to error?

- What is the relationship between whether, and/or how, physicians and other prescribers are taught to give V0 and the potential for error?

Strategies for minimising V0-related errors and harm

- What are the best practices to ensure appropriate face-to-face and/or telephone V0 use, communication, understanding and transcription?

- Would standardised V0 communication process based on the SBAR (Situation, Background, Assessment, Recommendation) or other structured communication methods significantly increase the accuracy of verbally reported clinical data, reduce the mishearing and misunderstanding of orders, ensure more consistent clarification of orders and/or improve initial order documentation and subsequent transcription?

- To what extent do VO represent workarounds used to avoid using CPOE systems to enter orders?

- To what extent do "inappropriate" V0 contribute to nurse job dissatisfaction because they are viewed as shifting workload from the prescriber to the nurse? 
or subsequently transcribing the $\mathrm{VO}$ into the medical record. Cases 5 and 6 in box 1 provide examples of such errors. Not shown in fig 1 is the final step in which the prescriber reviews, validates and signs the transcribed VO. Because this final check on the accuracy of a VO occurs only after the $\mathrm{VO}$ has been carried out, it is essential for VO to be correctly communicated, understood and transcribed.

It should be noted that the process and potential for error depicted in fig 1 are applicable to situations in which VO are used because of medical necessity (ie, during emergencies and procedures, or prescriber is not physically present) as well as for prescriber convenience. Although there are no empirical data that associate the use of VO with increased medical errors, as noted earlier it is widely believed that the use of $\mathrm{VO}$ in situations where they are not immediately necessary for patient care (ie, provider convenience) may contribute to avoidable errors and adverse events as well increasing workload for those receiving the orders. Unanswered are the questions of the nature and extent of the threat to patient safety posed by VO.

\section{POTENTIAL VO RESEARCH AGENDA}

Because of the paucity $\mathrm{VO}$ research, there is both a great need and opportunity for the development of a research agenda in this area. Conceptually it is useful to organise the broad range of questions needing further research under three categories: (1) Nature, Extent and Appropriateness of Current VO Use; (2) Nature, Extent and Causal Role of VO in Medical Error; and (3) Strategies for Minimising VO-Related Errors and Harm. Examples of specific research questions for each category are listed in box 2 .

\section{SUMMARY}

VO represent a commonly used and perceived as a potential threat to patient safety. Despite their common use and anecdotal evidence, there has been very little systematic study of the actual threat posed by their use. As a yet unstudied but common communication vehicle in healthcare, VO represent a very important and rich area for future research of direct relevance to improving patient care safety.

Funding: This work is supported by funding from AHRQ-THQIT Implementation \#1 UC1HS015196 and the University of Missouri Center for Health Care Quality.

Competing interests: None.

\section{REFERENCES}

1. West D, Levine S, Magra G, et al. Pediatric medication order error rates related to the mode of order transmission. Arch Pediatr Adolesc Med 1994;148:1322-6.

2. Kaplan JM, Ancheta R, Jacobs BR. Inpatient verbal orders and the impact of computerized provider order entry. J Pediatr 2006;149:461-7

3. Wakefield DS, Ward MM, Groath D, et al. Complexity of medication related verbal orders. Am J Med Qual 2008;23:7-17.

4. Shojania K, Duncan B, McDonald K, et al. Safe but sound: patient safety meets evidence-based medicine. JAMA 2002;288:508-13.

5. Chai K. 2004 \& 2005: patient safety goals and the impact on the JCAHO survey. Glendale: Cinahl Information Systems, 2005

6. Verbal orders. Right drug, wrong route, Nursing 2002;32:73

7. Dahl FC, Davis NM. A survey of hospital policies on verbal orders. Hosp Pharm 1990;25:443-7.

8. Joint Commission Resources. Verbal orders: strategies for proper use. Joint Commission Perspectives on Patient Safety, July 2003;3. http://www.jcrinc.com/ 4897/Mar07 (assessed 15 Mar 2007).

9. ECRI. Improving the safety of verbal orders. Patient Safety Advisor 2006;3:3-7.

10. National Quality Forum. Safe practices for better healthcare: a consensus report. http://www.jcrinc.com/5117/?query=Verbal\%200rders (accessed 5 Mar 2007).

11. Paparella S. Avoid Verbal Orders. J Emerg Nurs 2004;30:157-9.

12. Reason J. Human error: models of management. BMJ 2000;320:768-70.

13. Allison TT, Szeinbach SL, Schneider PJ. Perceived accuracy of drug orders transmitted orally by telephone. Am J Health-Syst Pharm 2005;62:78-83.

14. National Coordinating Council for Medication Error Reporting and Prevention. Recommendations to reduce medication errors associated with verbal medication orders and prescriptions adopted 20 February 2001, revised 24 February 2006.

15. Pennsylvania Patient Safety Authority. Sample policy on verbal/telephone orders http://www.psa.state.pa.us/psa/lib/psa/verbal_orders_toolkit/sample_policy_ _verbal_orders.pdf (accessed 5 May 2007).

16. Anon. Joint Commission Resources: Guidelines for accepting and transcribing verbal or telephone orders. The Source 2006;4:6-10.

17. Institute for Safe Medication Practices. Thalidomide and flutamide. ISMP Medication Alert 2004;21:29 January. http://www.ismp.org/Newsletters/acutecare/ articles/A1004Action.asp?ptr=y (accessed 30 Apr 2009)

18. Institute for Safe Medication Practices. Verbal order for "40 of K." ISMP Medication Alert 2002;17:3 October. http://www.ismp.org/Newsletters/acutecare/ articles/A4002Action.asp?ptr=y (accessed 30 Apr 2009)

19. Patient Safety Authority. Improving the safety of telephone or verbal orders. Pat Saf Advisor 2006;3. http://www.psa.state.pa.us/psa/lib/psa/press_releases/ 2006 june advisory final (2).pdf (accessed 4 May 2007).

20. United States Pharmacopeia. Practitioners' Reporting News, Current Confusion Between Amiodarone and Amrinone, United States Pharmacopeia 28 January 2000 http://www.usp.org/hqi/practitionerPrograms/newsletters/ practitionerReportingNews/archive/prn642000-01-28.html (accessed 30 Apr 2009).

21. Institute for Safe Medication Practices. ISMP Medication Alert. Preventing magnesium toxicity in obstetrics. http://www.ismp.org/Newsletters/acutecare/ articles/20051020.asp?ptr=y (accessed 30 Apr 2009).

22. Agency for Healthcare Research and Quality. Getting to the Root of the Matter AHRO Web M\&M Case \& Commentary, June, 2005. http://www.webmm.ahrq.gov/ case.aspx?caselD=98qsearchStr=\%22verbal+order\%22 (accessed 30 Apr 2009). 